\title{
A Building Performance Indicator Ontology: Structure and Applications
}

\author{
Ardeshir Mahdavi, Dawid Wolosiuk \\ TU Wien, Vienna, Austria
}

\begin{abstract}
This paper introduces a proposal for a comprehensive ontology of Building Performance Indicators (BPI). To establish this ontology, we utilize an extensive review of BPIs in thermal, air quality, visual, and acoustical domains. For each domain, categorical classification schemes are established first. We then list, for each category, illustrative instances of common BPIs. Subsequently, the ontology's structural core is introduced, together with a systematic specification of the attributes of building performance variables. To demonstrate the general applicability of the ontology, it is tested against a diverse sample of BPIs. Finally, we exemplify the utility of the ontologically structured BPI information with visualization application as a case in point.
\end{abstract}

\section{Introduction}

Building performance assessment procedures typically make use of a large number of building performance indicators (BPI), involving multiple domains, aspects, and degrees of resolution. Use cases of BPIs are diverse. These include, compliance demonstration with building code requirements, specification of building attributes in certificate-type documents, as well as comparison and ranking of building design alternatives (Mahdavi and Taheri, 2018). However, despite the pervasive use of BPIs, there have been very few attempts to compose an explicit BPI ontology. The present contribution addresses this gap via the introduction of a systematic and comprehensive BPI ontology, which can offer multiple benefits. As such, ontologies can help structure the conceptual and semantic constituents of a domain and thus improve the efficiency of communication processes and developmental work therein. Whether obtained via measurement or simulation, the values of ontologically well-formed BPIs can support visualization, optimization, and other decision support applications. A versatile BPI ontology can add to the clarity of building performance requirements specifications, advance the understanding of building performance principles in educational and training settings, and provide a solid foundation for the development of all-purpose data visualization engines (Mahdavi et al., 2016; 2017; 2018; 2005).

The development of ontology relied on an extensive review of common BPIs in thermal, air quality, visual, and acoustical domains. For each domain, categorical classification schemes were established and, for each category, concrete instances of common BPIs were considered. The ontology's structural core involves a systematic specification of the generic attributes of building performance variables. The general applicability of the ontology was demonstrated in that it was tested against a sample of BPIs from different domains. Moreover, we explored the potential of the ontology in organizing and processing information based on the example of performance data visualization.

\section{Building Performance Indicators}

The performance of buildings can be characterized through an extensive list of BPIs pertaining to multiple domains. These span from ones that assess strictly technical systems performance, to those that are relevant to the "habitability" aspects of the building, including human health, comfort, and satisfaction (Mahdavi, 1998; 2011). A recent effort attempted to compile, review, and categorize a large number of BPIs (Constantino, 2017). These were organized into topical domains, including energy efficiency, hygro-thermal performance, thermal comfort, air quality, visual environment, and acoustical environment. Based on this effort, a general scheme of BPI categories was proposed as per Figure 1. Therein, the main domain categories are mapped to relevant subcategories, exemplified by illustrative indicator instances.

\section{Indicators and variables}

The aforementioned review of BPIs facilitated a key observation. Despite their diversity, most indicators can be either directly mapped back to a measurable variable, or are derived based on algorithmic manipulation of one or more of such variables. As such, the essential attributes of a BPI are similar to (or can be derived from) those applicable to measurable variables. Frequently, a primary (directly measured) variable, such as indoor air temperature itself, can act as a BPI (in this case, in the thermal performance category).

Aggregation (or other types of statistical treatment) of the values of such a variable over time and space can lead to more complex BPI definitions. Likewise, multiple, variously measured, calculated, estimated, or simulated variables can be synthetized into a compound BPI. A classic example of such a compound indicator would be PMV (Predicted Mean Vote), whose definition requires the inclusion and algorithmic processing of values of variables pertaining to environmental conditions (air 


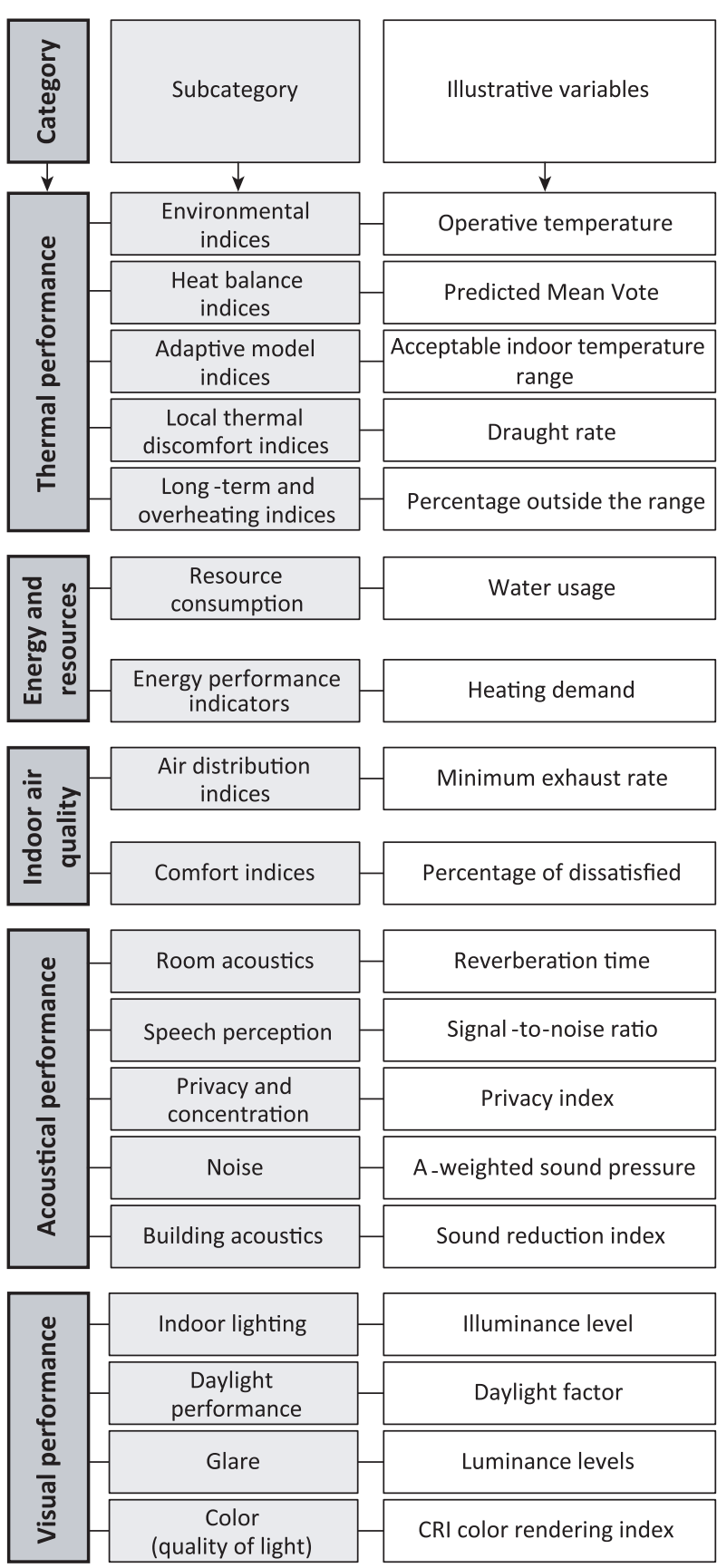

Figure 1: An overview of five building performance domains with subcategories and illustrative performance indicators examples (see Constantinou (2017) for more details).

temperature, radiant temperature, air flow speed, air relative humidity) and occupants' personal factors (metabolic rate, clothing resistance). Guided by this observation and our previous experiences with performance data ontologies, we suggest that the essential ontological schema for building performance indicators can be obtained vis slight modifications to a previously introduced ontology of the primary building performance variables (Mahdavi and Taheri, 2018) including the Agent ID item and revisions in the Notes category).

\section{Ontological schema for building performance indicators}

Utilizing the general map of indicators presented in Figure 1, and considering additional aspects and requirements of measurable performance variables (Mahdavi and Taheri, 2018), a schema is proposed to capture the essential, ontologically relevant characteristics of building performance indicators (see Table 1). This schema can be shown to fulfill key requirements of both measurable performance variables and BPIs, be those primary variables with actual values or compound variables with derived values. Each variable under consideration is mainly identified by its domain category and subcategory. The primary content of the schema is the variable's value. It carries a number of attributes and properties. The variable's type mainly specifies if its values are continuous or discrete. Each variable value is assumed to have a magnitude and, in certain cases, a direction vector. A relevant unit should be specified for valid data interpretation and processing. Variable's category determines a number of additional properties that are specified in three domains. Spatial domain properties allow to associate a variable with a specific point in a Cartesian coordinate system or with a topologically specified location (for instance, via a room tag). Temporal domain properties can be expressed in the schema via a time stamp (e.g., for a sensor reading).

Table 1: General schema for BPIs, modified based on Mahdavi and Taheri (2018).

\begin{tabular}{|c|c|c|c|}
\hline Category & & & \\
\hline Sub-categ & & & \\
\hline & Name & & \\
\hline & & Type & \\
\hline & & Magnitude (size) & \\
\hline & & Direction (vector) & \\
\hline & & Unit & \\
\hline & & & Point \\
\hline & & & Plane \\
\hline & & Snatioldomain & Volume \\
\hline & & Spatıal domain & Topological ref. \\
\hline & Volur & & Aggregation method \\
\hline & Value & & Grid size \\
\hline Variable & & & Time stamp \\
\hline & & & Duration \\
\hline & & Temporal & Time step \\
\hline & & & Aggregation method \\
\hline & & & Range \\
\hline & & Frequency & Band (filter) \\
\hline & & domain & Weighting \\
\hline & & & Aggregation method \\
\hline & Agent & & ID \\
\hline & & Data sources & Category \\
\hline & Notes & Data sources & ID/name \\
\hline & & $\begin{array}{l}\text { Derivation } \\
\text { method }\end{array}$ & $\begin{array}{l}\text { Details (formula, } \\
\text { link, etc.) }\end{array}$ \\
\hline
\end{tabular}


A time step denotes recurrent temporal intervals to which measured or simulation data could be assigned (e.g., hourly heating loads). Duration denotes the overall time frame to which a given variable value corresponds (e.g., annual cooling load). As such, time step and grid size can specify the discretization resolution of pertinent temporal and spatial continua. The Frequency domain attributes are relevant to measured or simulated values that display wave characteristics (e.g., light/radiation, sound).

If relevant, an agent ID attribute can be used to assign a given variable to a specific entity. Notes category pertains to additional relevant information addressing, for instance, the indicators' derivation background. In case of a monitored variable, notes could include a unique ID of a meter or sensor. In case of derived and compound indicators, notes could point to the pertinent computational procedure, such as applicable formulas, algorithms, and associated links and resources.

\section{Application of the proposed schema}

Three exemplary variables from different domains were selected pars pro toto to illustrate potential and robustness of the ontology (see Table 2). Thereby, categories, subcategories, and attributes of a given variables are indicated.

\section{From data sources to applications}

Data or datasets consisting solely of an array of numbers without any additional descriptors cannot meet the requirements of the down-stream applications. Once descriptive and contextual content is added, data usability and scientific potential increases. Description of content, context, and structure should be an integral part of any dataset. Files containing data in a tabular form (e.g., CSV file format) is commonly accompanied by a description file, or such details are stored in the header of a file. This is not a very effective way of contextualizing data, especially when it is a part of a larger dataset. Data stored in this form is "flat" in nature. The bulk of context and the main hierarchy remain only on a descriptive level and must be interpreted. The schema of the proposed BPI ontology displays a highly hierarchical nature that is supposed to capture its complexities. It would be beneficial to maintain clear structure and contextual details to support further information processing.

Considering the aforementioned observations, the selection of a proper data container for the said ontology is crucial for developing its application potential. Such a container would have to be capable of holding hierarchically structured sets of data together with attributes pertaining to multiple categories, subcategories, and variables. There may be multiple solutions that could meet the above requirements. However, after a general review and a number of exploratory trails, we opted for the HDF (Hierarchical Data Format) file format, specifically its most recent version HDF5 (HDF group, 2018) as the container for ontologically structured data. This file format was designed by National Center for Supercomputing Applications (NCSA) to support platform independent data storage and manipulation
(NASA, 2018). It was developed primarily as a scientific data storage format. Hence, one of the most important properties of the HDF5 file is its self-describing nature. It allows for efficient data exchange and makes archived data more accessible, mainly due to the transparent hierarchical structure, elements attribution, and annotation capacity. As a result, HDF5 data can be effectively accessed and queried by various applications, including those in the performance assessment domain.

Figure 2 provides a schematic overview of the proposed structured path from "low-level" primary performancerelated data to various "high-level" performance-relevant applications. Building related performance data can originate from various sources. These include, for example, monitoring data (indoor conditions, energy use, system states) as well as calculation or simulation data (both input and output data). All these sources naturally come in different forms, formats, or resolutions (i.e. storage file types, internal structure, notation conventions, etc.). A series of procedural steps needs to be taken in

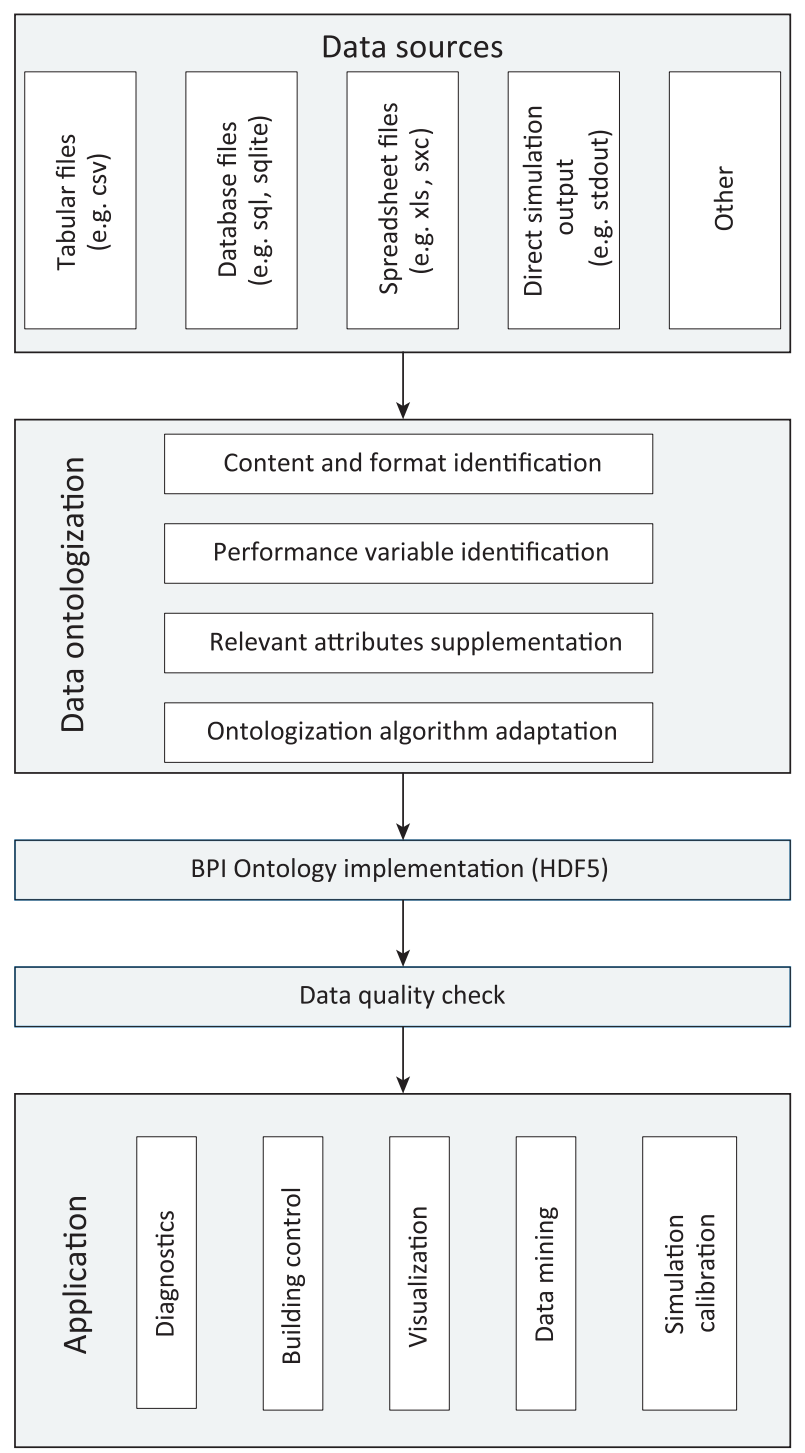

Figure 2: Schematic overview of the proposed structured path from "low-level" primary performance-related data to various "high-level" applications. 
Table 2: Illustrative representation of three exemplary BPIs following the structure of the proposed ontology, modified based on Mahdavi and Taheri (2018).

\begin{tabular}{|c|c|c|c|c|c|c|}
\hline \multicolumn{4}{|c|}{ Category } & Energy and resources & $\begin{array}{l}\text { Thermal } \\
\text { performance }\end{array}$ & $\begin{array}{l}\text { Visual } \\
\text { performance }\end{array}$ \\
\hline \multicolumn{4}{|c|}{ Subcategory } & $\begin{array}{l}\text { Energy performance } \\
\text { indicator }\end{array}$ & $\begin{array}{l}\text { Heat balance } \\
\text { indices }\end{array}$ & Indoor lighting \\
\hline \multirow{23}{*}{$\begin{array}{l}\frac{0}{\pi} \\
\frac{\pi}{\tilde{\sigma}} \\
\stackrel{\nabla}{>}\end{array}$} & Name & & & Heating Load & Predicted Mean Vote & Illuminance levels \\
\hline & \multirow{18}{*}{ Value } & Type & & Derived, Ratio & Derived, Interval & Primary, Ratio \\
\hline & & Magnitude & & 50 & -0.13 & 463 \\
\hline & & Direction & & - & - & {$[0,0,1]$} \\
\hline & & Unit & & kWh.m ${ }^{2}$ & - & $1 \mathrm{x}$ \\
\hline & & \multirow{6}{*}{$\begin{array}{l}\text { Spatial } \\
\text { domain }\end{array}$} & Point & - & {$[1.00,4.50]$} & {$[1.00,4.00,0.85]$} \\
\hline & & & Plane & - & - & - \\
\hline & & & Volume & Building A & - & - \\
\hline & & & $\begin{array}{l}\text { Topological } \\
\text { reference }\end{array}$ & Building A & Office_012 & Office_012 \\
\hline & & & $\begin{array}{l}\text { Aggregation } \\
\text { method }\end{array}$ & - & - & - \\
\hline & & & Grid size & - & - & - \\
\hline & & \multirow{4}{*}{$\begin{array}{l}\text { Temporal } \\
\text { domain }\end{array}$} & Time stamp & - & $\begin{array}{l}\text { 11.06.2018 } \\
09: 00: 00\end{array}$ & $\begin{array}{l}01.12 .2018 \\
12: 40: 00\end{array}$ \\
\hline & & & Duration & Annual & - & - \\
\hline & & & Time step & 1 hour & 1 hour & $5 \mathrm{~min}$ \\
\hline & & & $\begin{array}{l}\text { Aggregation } \\
\text { method }\end{array}$ & $\begin{array}{l}\text { Arithmetic } \\
\text { summation }\end{array}$ & $\begin{array}{l}\text { Arithmetic } \\
\text { average }\end{array}$ & $\begin{array}{l}\text { Arithmetic } \\
\text { average }\end{array}$ \\
\hline & & \multirow{4}{*}{$\begin{array}{l}\text { Frequency } \\
\text { domain }\end{array}$} & Range & - & - & - \\
\hline & & & Band & - & - & - \\
\hline & & & Weighting & - & - & - \\
\hline & & & $\begin{array}{l}\text { Aggregation } \\
\text { method }\end{array}$ & - & - & - \\
\hline & Agent & & ID & - & Occupant_4 & - \\
\hline & \multirow{3}{*}{ Notes } & \multirow{2}{*}{$\begin{array}{l}\text { Data } \\
\text { sources }\end{array}$} & Category & Simulation & Sensor & Sensor \\
\hline & & & ID/name & Sim_20180729_1 & $\begin{array}{l}\text { Tem_3, Rh_3, airV_1, } \\
\text { occ4_met, occ4_clo, } . .\end{array}$ & lux_4 \\
\hline & & $\begin{array}{l}\text { Derivation } \\
\text { method }\end{array}$ & & - & https://www.iso.org/... & - \\
\hline
\end{tabular}

order to structure the data in conformance with the proposed ontological schema. Therefore, file or input content needs to be identified and variables need to be categorized and supplemented with relevant attributes. This step requires intensive user input as most of the attributes pertaining to different domains might not be included in the original data set, or might only exist as a separate dataset description. The required data enrichment requires additional insight into the data and some measure of expert knowledge. Ideally, the related process could be supported via an interface that allows for the recognition of common data storage formats and assignment of 
pertinent variable attributes. Such an interface would not be able to exhaustively cover all intricacies of diverse data sources. For the purpose of the present effort, we developed an algorithm that that can translate selected input data formats and supplemented attributes into the hierarchical structure of HDF5 file.

Before building performance data is further processed by interested application, the integrity and quality of data should be examined. Of course, ontologically structured data in HDF5 files might have undergone some quality checking operations in the preceding steps of the process. Nonetheless, the proposed data quality check step in our scheme (see Figure 2) fosters the confidence in the reliability of the applications input data pool and their final results.

The majority of applications such as diagnostics, building control, or data mining, go through a logical query process to extract specific segments of data such that certain conditions are satisfied. We believe that the proposed structured process (involving "ontologization" and efficient HDF5-supported data storage and query) can provide an effective platform to meet the requirements of various applications such as those listed above. To illustrate this point, we focus, in the following section, on one of the most powerful data analysis methods, namely (performance) data visualization.

\section{Visualization as an illustrative case in point}

Data for the ontology implementation and application testing are obtained from multiple sensors and meters installed at the office space in a university building (TU Wien) in Vienna, Austria. Specifically, 116 unique variables observed over a period of 3 years are stored as distinct database files. Each performance variable is identified and supplemented with all known relevant attributes. As mentioned before, to support this process, a workflow was developed whereby attributes for all variables are collected in terms of a single tabular file for seamless implementation in HDF5 file.

To illustrate the utility and effectiveness of the wellformed semantically enriched data in HDF5 format, two demonstrative case studies are considered below. These address the potential for data visualization and interpretation. The first example pertains to (overhead) illuminance levels (hourly values) measured in an office area of the aforementioned university building over a whole year period (2017). The second example explores the dependency of measured indoor air temperatures in an open plan office area (in the same building) on the outdoor air temperatures.

The implementation process involved utilization of different programming environments, to demonstrate and verify universal support options for HDF5 format. The MATLAB software as well as the Python programming environment were used to produce the visualizations. The former has built-in functions that support creation, manipulation, and exploration of HDF5 files. The latter offers a library that provides a similar functionality. In both cases, input data for the visualizations was extracted from the said HDF5 file using available routines. Subsequently, the extracted data was processed locally to produce the final visual representations as detailed below. Regarding the first case study, Figure 3 entails a visualization of the measured illuminance levels in terms of a tile map. In case of the second case study (indooroutdoor temperature relationship), it was hypothesized that, due to the effect of thermal mass and associated temporal delay, indoor air temperatures may display a higher correlation with outdoor temperature measured at an earlier instance. We thus investigated the correlation between indoor temperature with outdoor temperature measured 30, 60, 90, 120, 150, and 180 minutes earlier (see Figure 4). This analysis suggest that the highest correlation involves a two-hour time shift between measured indoor and outdoor temperatures. Figure 5 displays this correlation graphically.

For both of the above cases logical queries were formulated to satisfy specific combinations of categorical, spatial, and temporal criteria. Specifically, in the first case study, "overhead illuminance", "seminar room", and "year 2017" were defined as variable name, spatial attribute, and temporal constraint respectively. In the second example: "indoor/outdoor temperature", "office area", "21.06.2017 - 22.09.2017" were defined as variable name, spatial attribute (only for indoor temperature), and temporal constraint respectively.

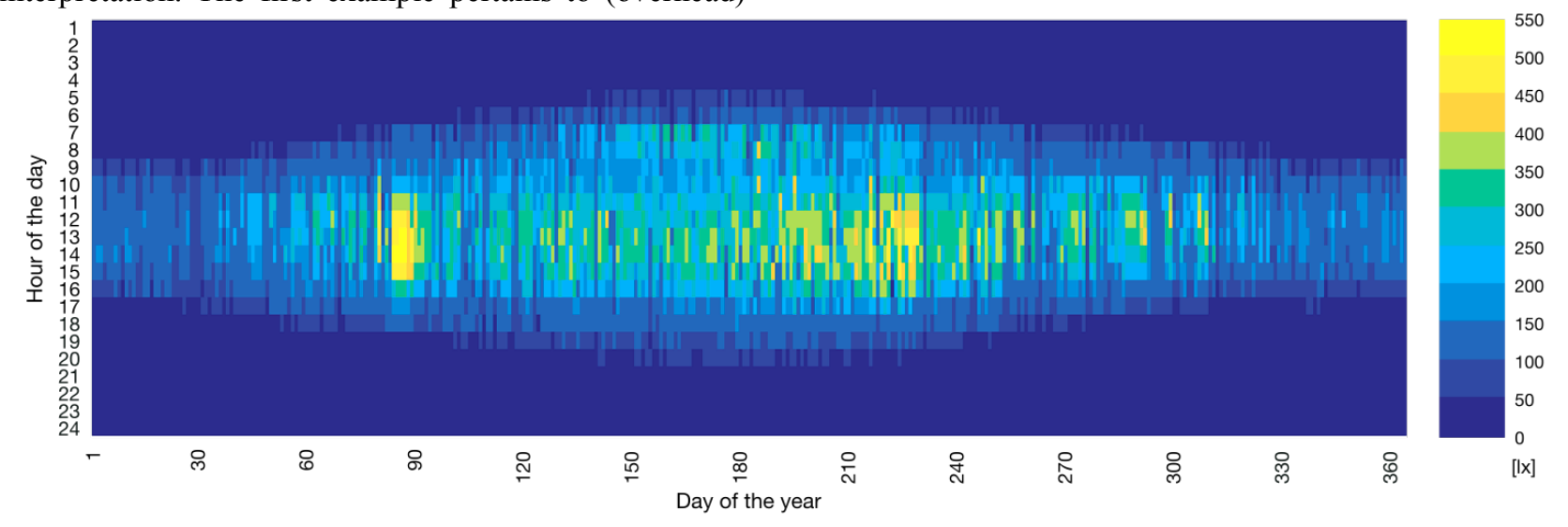

Figure 3: A tile map visualization of measured overhead illuminance levels in an office area. 


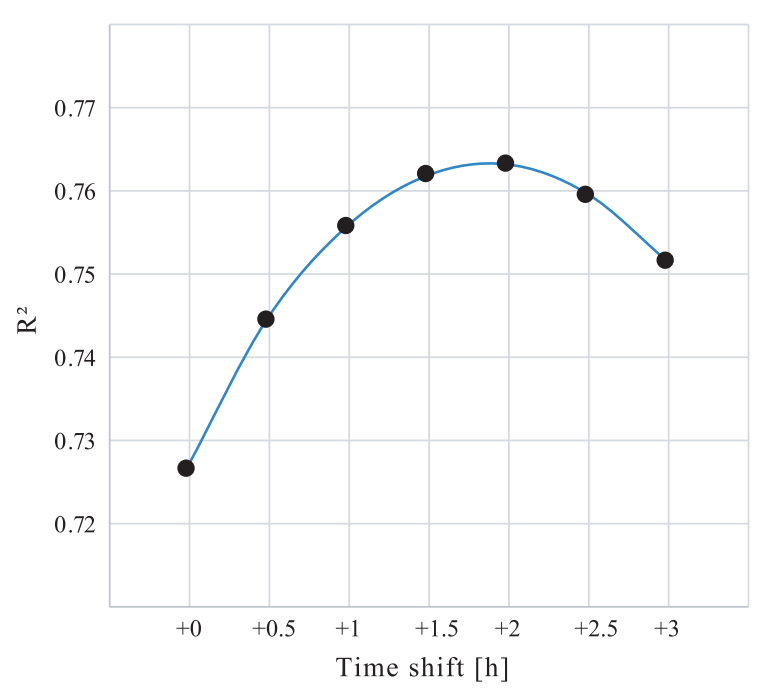

Figure 4: Coefficient of determination between measured indoor air temperatures in an office area and earlier measurements of outdoor temperature (from half an hour to three hours before).

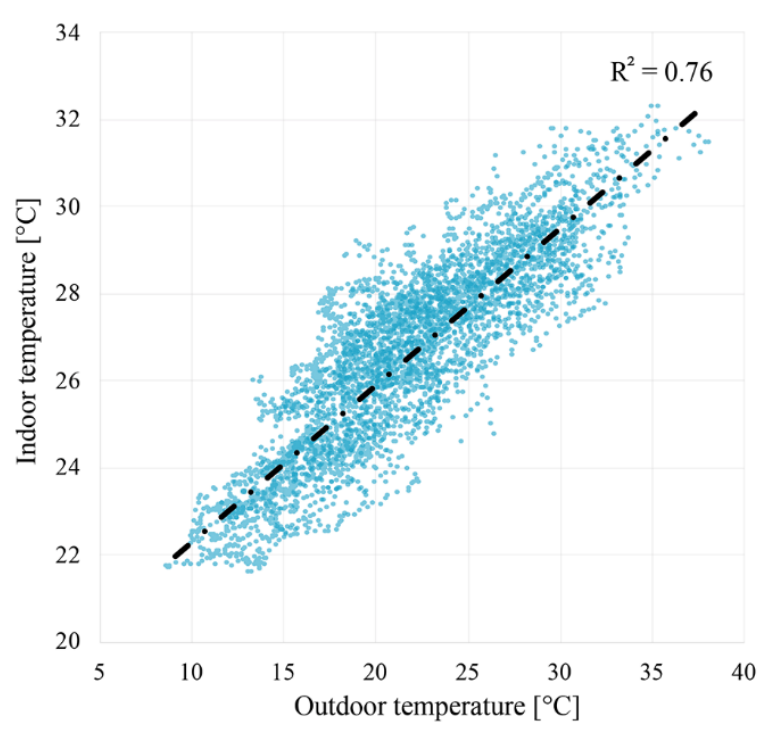

Figure 5: Illustration of the correlation between measured indoor and (two-hour shifted) outdoor temperatures.

\section{Conclusion}

Building upon a previous comprehensive survey of common building performance indicators as well foundational ontological work on monitoring data, we presented a respective systematic ontological schema. This schema is shown to capture key features of a wide range of building performance variables in multiple domains. Furthermore, we described a standard workflow whereby "low-level" primary performance-related data is "ontologized" and placed in a data container to enable further utilization in various "high-level" performancerelevant applications.
For illustration purposes, we demonstrated the robustness and utility of the ontology and the proposed data processing workflow using data visualization application as a case in point. Ongoing and future studies explore further applications. These include, but are not limited to, automated data quality examination, building operation optimization, performance data mining, simulation model calibration, performance-based contracting, and automated performance compliance checking.

\section{Acknowledgement}

The authors would like to acknowledge the support provided by Matthias Schuss and Josef Lechleitner toward the collection and processing of the data used in this paper.

\section{References}

Mahdavi, A., Taheri, M. (2018). A building performance ontology. Proceedings of ECPPM 2018, 385-390.

Mahdavi, A. (1998). Steps to general theory of habitability, Human Ecology Review 5(1).

Mahdavi A. (2011). People in Building Performance Simulation. In Hensen, J.L.M., Lamberts R. Building Performance Simulation for Design and Operation. Taylor \& Francis Group. New York (USA).

Constantinou, N. (2017). A comprehensive multi-domain building performance indicator catalogue. Master thesis. Department of Building Physics and Building Ecology, TU Wien. Vienna (Austria).

Mahdavi, A., Taheri, M., Schuss, M., Tahmasebi, F., and Glawischnig, S. (2018). Structured Building Data Management: Ontologies, Queries, and Platforms. Exploring Occupant Behavior in Buildings. Publisher: Springer. Editors: Andreas Wagner, William O'Brien, Bing Dong. DOI: 10.1007/978-3-319-61464-9_10.

Mahdavi, A., Bachinger, J., and Suter, G. (2005). Toward a unified information space for the specification of building performance simulation results. In: Building Simulation 2005. 9th International IBPSA Conference, Aug. 15-18, Montreal (Canada). Editors: I. Beausoleil-Morrison, M. Bernier. p.671-676.

Mahdavi, A., Taheri, M. (2017). An Ontology for Building Monitoring. Journal of Building Performance Simulation. 10(5-6), 499-508. DOI:10.1080/19401493.2016.1243730.

Mahdavi, A., Glawischnig, S., Schuss, M., Tahmasebi, F., and Heiderer, A. (2016). Structured Building Monitoring: Ontologies and Platform. Proceedings of ECPPM 2016: The 11th European Conference on Product and Process Modelling. Limassol (Cyprus)

The HDF Group, (2018). Hierarchical Data Format Version 5 (HDF5). https://www.hdfgroup.org/

NASA, (2018). Hierarchical Data Format. https://eosweb.larc.nasa.gov/sites/default/files/tools/h df.pdf 